\title{
Tinnitus in Temporomandibular Joint Disorders: Is it a Specific Somatosensory Tinnitus Subtype?
}

\author{
Giuseppe Alberto Maria Algieri \\ Alessandra Leonardi \\ Paolo Arangio \\ Valentino Vellone \\ Carlo Di Paolo \\ Piero Cascone
}

\begin{abstract}
The most significant otologic symptoms, consisting of ear pain, tinnitus, dizziness, hearing loss and auricolar "fullness", generally arise within the auditory system, often are associated with extra auricolar disorders, particularly disorder of the temporo-mandibular joint. In our study we examined a sample of 200 consecutive patients who had experienced severe disabling symptom. The patiens came to maxillofacial specialist assessment for temporomandibular disorder. Each patient was assessed by a detailed anamnestic and clinical temporomandibular joint examination and they are divided into five main groups according classification criteria established by Wilkes; tinnitus and subjective indicators of pain are evaluated. The results of this study provide a close correlation between the joint pathology and otologic symptoms, particularly regarding tinnitus and balance disorders, and that this relationship is greater the more advanced is the stage of joint pathology. Moreover, this study shows that TMD-related tinnitus principally affects a younger population (average fifth decade of life) and mainly women (more than 2/3 of the cases). Such evidence suggests the existence of a specific tinnitus subtype that may be defined as "TMD-related somatosensory tinnitus".
\end{abstract}

Keywords: tinnitus; trigeminal nerve; temporomandibular joint disorders

Odontostomathologic and Maxillo- Facial Science Department, "Sapienza" University of Rome, Via Caserta 6,00161, Rome, Italy. Email: algierig@yahoo.it, leonardia@ yahoo.it, arangiop@yahoo.it, valentino.vellone@gmail.com, diapaoloc@yahoo.it, casconep@yahoo.it

Institution: Odontostomathologic and Maxillo- Facial Science Department, "Sapienza" University of Rome, Via Caserta 600161, Rome, Italy

Send correspondence to:

Valentino Vellone

Via Teano 35, Latina, Italy. +39 3289489538. E-mail: valentino.vellone@gmail.com

Paper submitted to the ITJ-EM (Editorial Manager System) on August 23, 2016;

and accepted on December 12, 2016 


\section{INTRODUCTION}

The most significant otologic symptoms, consisting of ear pain, tinnitus, dizziness, hearing loss and auricular "fullness", generally arise within the auditory system, potentially at any level but, in clinical practice, they are often associated with extra auricular disorders, particularly disorders of the nearby temporomandibular joint (TMJ). In 1934, Costen ${ }^{1}$ sensed the possibility of a causal link between tinnitus and temporomandibular disorder (TMD), describing a series of related symptoms that characterized the well-known syndrome. Over the years, the increasing knowledge of anatomy and pathophysiology has made outdated the concept of "Costen's syndrome". In the '50s, mainly thanks to Schwartz, new interpretative models aimed to emphasize more muscular and emotional factors were proposed.

Between the '60s and '70s Moulton', Laskin'3 and Dworkin ${ }^{4}$ suggested an etiopatogenetic and pathophysiological interpretation in which the occlusal, muscular and psychological components compete with each other in a multi-factorial model. Since the early ' 90 s a number of research streams shed new light on the neurophysiopathological aspects of craniofacial pain, the true novelty being the concept of "chronic pain from deafferentation", triggered by peripheral nerve damage, albeit limited in time, and maintained by phenomena related to neuroplasticity. Despite all this, it remains to be clarified with certainty the relationship between the pathogenesis of tinnitus and $\mathrm{TMJ}^{5-6}$. The aim of the present study is to give a contribution on the relationship between tinnitus and TMD, emphasizing the importance of this symptom in disorders of the TMJ, and a suggestion for a multidisciplinary approach including ENT, gnathologist, and maxillofacial surgery evaluations.

\section{MATERIALS AND METHODS}

In the present study we considered a sample of 200 consecutive patients sent from ENT specialists, after excluding otologic causes, to maxillofacial specialist for TMD assessment from January to June 2013. All patients had experienced severe disabling tinnitus which influenced their lives. The sample consists of 168 females (84\%) and 32 males (32\%), with a ratio $\mathrm{F}: \mathrm{M}=5.25: 1$, aged between 13 and 79 years (mean age 44.1). Each patient was assessed by a detailed anamnestic, clinical examination with a specific chart filled for patients affected by TMD. Moreover, a magnetic resonance (MR) imaging that allows to determinate articular disk location or meniscus, its morphologic features and its location relative to the condyle in both closed- and open-mouth positions was request.

Subjective indicators of pain (temporomandibular joint pain, headache, neck pain and shoulder pain) was evaluated using visual analogue scales (VAS, range: 0 to $100)$ to indicate the numerical value in terms of self-valued severity, whereas tinnitus and balance disorders/dizziness were assessed with 2 questionnaire, respectively, tinnitus handicap inventory $(\mathrm{THI})^{7}$ and dizziness handicap inventory $(\mathrm{DHI})^{8}$.

We used classification criteria established by Wilke ${ }^{9}$ (Table 1), on the basis of which the patients in the study were divided into five main groups:

Group I (TMD early stage): 11 patients $(5.5 \%$ of the total sample);

Group II (TMD Early/intermediate stage): 67 patients $(33.5 \%$ of the total sample, which is the largest group);

Table 1. Clyde H Wilkes classification.

\begin{tabular}{ll}
\hline Group & Classification \\
\hline Early stage & Clinical: no significant mechanical symptoms, othe \\
& than reciprocal clicking (early in opening movement, \\
& and soft in intensity). No pain and limitation of moti \\
& Radiologic: slight forward displacement, good \\
& anatomical contour of disk, and normal tomograms \\
& Surgical: normal anatomical form, slight anterior \\
& displacement, and passive incoordination (clicking \\
& demonstrable
\end{tabular}

intermediate tenderness and related temporal headaches, beginning stage major mechanical problems, increase in intensity of clicking sounds, joint sounds later in opening movements, and beginning transient subluxations or joint catching and locking.

Radiologic: slight forward displacement, slight thickening of posterior edge or beginning anatomical deformity of disk, and normal tomograms.

Surgical: anterior displacement, early anatomical deformity of disk( slight to mild deformity thickening of posterior edge) and well defined central articulating area.

Intermediate Clinical: multiple episodes of pain, joint tenderness, stage temporal headaches, major mechanical symptomstransient catching, locking and sustained locking (closed locks) restriction of motion and difficulty (pain) with function.

Radiologic: anterior displacement with significant anatomical deformity/prolapsed of disk (moderate to marked thickening of posterior edge) and normal tomograms.

Surgical: marked anatomical deformity with displacement, variable adhesions (anterior, lateral, and posterior recesses), and no hard tissue change.

Intermediate/ Clinical: characterized by chronicity with variable late stage and episodic pain, headaches, variable restriction of motion, and undulating course.

Radiologic: increase in severity over intermediate stage, abnormal tomograms, and early to moderate degenerative remodeling hard tissues changes. Surgical: increase on severity over intermediate stage, hard tissue degenerative remodeling changes of both bearing surfaces, ostheophytic projections, multiple adhesions (lateral, anterior and posterior recesses) and no perforation disk attachment.

Late stage Clinical: Characterized by crepitus on examination scraping, grating, grinding symptoms variable and episodic pain, chronic restriction of motion, and difficulty of function.

Radiologic: anterior displacement, perforation with simultaneous filling of upper and lower compartments, filling defects, gross anatomical deformity of disc and hard tissues, abnormal tomograms as described, and essentially degenerative arthritic changes. Surgical: gross degenerative changes of disc and hard tissues, perforation of posterior attachments, erosions of bearing surfaces, and multiple adhesions equivalent to degenerative arthritis (sclerosis, flattening, anvilshaped condyle, ostheopytic, projections, and subcortical cystic formation. 
Group III (TMD Intermediate stage): 58 patients (29\% of the sample);

Group IV (TMD Intermediate/late stage): 53 patients (26.5\% of the sample);

Group V (TMD Late stage): 11 patients (5.5\% of the sample).

The main data are summarized in Figure 1.

\section{RESULTS}

The study was carried out on a sequential sample of patients who all received maxillofacial examination for the evaluation of temporo-mandibular joint disease. All patients went through anamnestic, clinical and instrumental evaluation in order to assess their stage according to the Wilkes classification. In the analysis of the set of symptoms associated with TMD we mostly focused on the incidence of otologic symptoms, namely, tinnitus and balance disorders. The ratio of these two otologic symptoms was substantially comparable in the sample, with tinnitus present in $60 \%$ of cases, and impaired balance / dizziness in $59.5 \%$ (Figure 1). We noticed that the tinnitus was more frequently associated with increasing severity of the TMD (Figure 2), from an incidence of $54.5 \%$ in the first group (early stage) to $90.9 \%$ in the fifth (late stage). Another important consideration is that greater the severity of the joint disease more the THI grade increases: from an average of 18.2 to 44.5 in the late stage.

Group I (TMD early stage): 11 patients (5.5\% of the total sample), 6 with tinnitus, accounting for $54.5 \%$ of the group (4 bilateral and 2 unilateral), and 5 with dizziness (45.4\%); THI scores of $18.2 \%$.

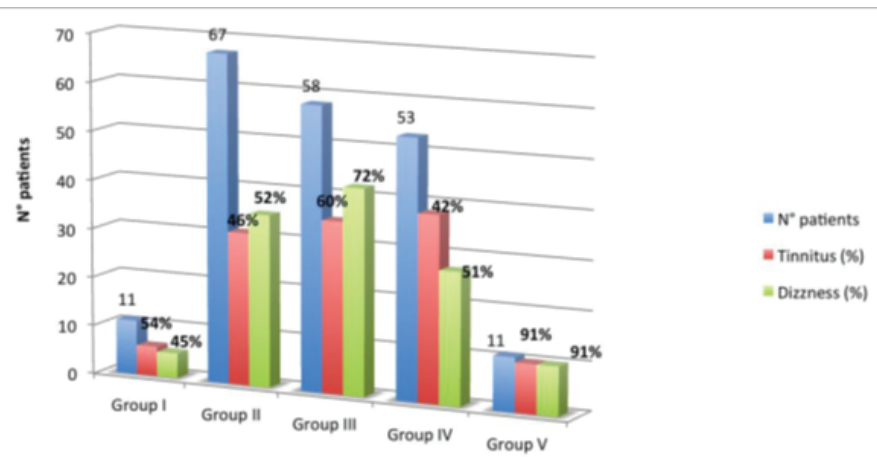

Figure 1. Classification of patientst according to the classes of Wilkes and\% incidence of tinnitus and dizziness for the group.

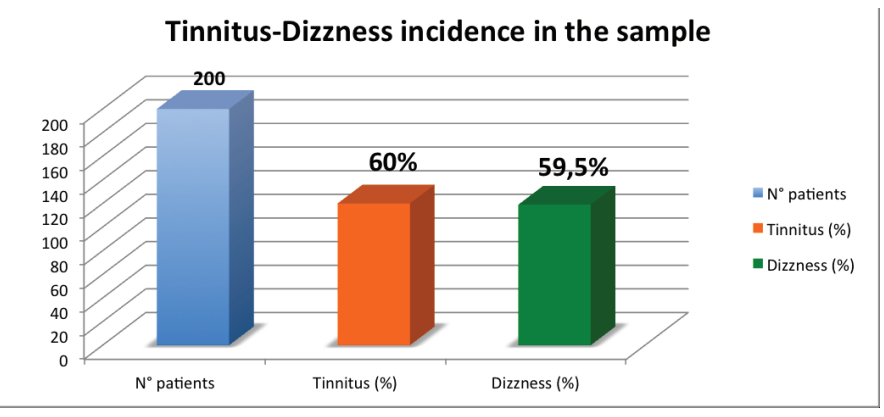

Figure 2. Incidence of tinnitus and vertigo in the total study sample.
Group II (TMD Early/intermediate stage): 67 patients $(33.5 \%$ of the total sample, which is the largest group), 31 with tinnitus, accounting for $46.2 \%$ of the group (17 bilateral, 14 unilateral) and 35 with dizziness (52.2\%); THI scores of $22.8 \%$.

Group III (TMD Intermediate stage): 58 patients (29\% of the sample), 35 with tinnitus, accounting for $60.3 \%$ of the group (26 bilateral, 9 unilateral) and 42 with dizziness (72.4\%); THI scores of $34.8 \%$.

Group IV (TMD Intermediate/late stage): 53 patients (26.5\% of the sample), 38 with tinnitus, accounting for $71.7 \%$ of the group (28 bilateral, 10 unilateral) and 27 with dizziness $50.9 \%$ ); $\mathrm{THI}$ scores of $35.7 \%$.

Group V (TMD Late stage): 11 patients (5.5\% of the sample), 10 with tinnitus, accounting for $90.9 \%$ (8 bilateral, 2 unilateral) and 10 with dizziness (90.9\%); THI scores of $44.5 \%$.

\section{DISCUSSION}

Tinnitus is classically defined as the perception of sound or a conscious experience in which an individual hears a noise that originates inside the head, in the absence of any external sound source. In addition, across the literature, tinnitus is considered as an extremely heterogeneous condition. Most authors distinguish the subjective and objective tinnitus. The objective tinnitus (or extra- auditory), rarer, should be, by definition, potentially audible even by the examiner. As a matter of fact, any tinnitus is considered objective if originated outside of the auditory system (muscular noise, clicks, or vascular pulse noise), regardless of its auscultation. Subjective tinnitus (or intra-auditory), similar to auditory hallucinations (schizophrenia or hallucinogenic drug), is a real "ghost phenomenon." By definition tinnitus is neither a syndrome or a disease, but a symptom, experienced by the patient as unilateral, bilateral or without specific reference to the side, associated with a wide variety of possible causes, and in particular:

Otological causes: noise-induced hearing loss, presbycusis, otosclerosis, otitis externa and media (acute and chronic), earwax or ear canal stenosis by foreign body, Meniere's disease, sudden hearing loss and other causes of hearing loss;

Neurological causes: cranio-cervical trauma, including whiplash injuries, multiple sclerosis, acoustic neuroma of the $8^{\text {th }}$ cranial nerve, other tumors of the pontocerebellar angle;

Infectious-inflammatory causes: otitic processes, the sequelae of Lyme syndrome, meningitis, tertiary syphilis and other infectious-inflammatory or inflammatory processes affecting the auditory components at any level;

Toxic causes, particularly drugs consumption: salicylates, aminoglycoside antibiotics, loop diuretics, benzodiazepines, tricyclic antidepressants, as well as some chemotherapy; 
Systemic causes: hypertension, polycythemia, sickle cell anemia, renal failure, allergic diathesis, cycle estrogen-progestin.

The causes that are most frequently associated with the occurrence of permanent or at least long-term tinnitus (Oregon Tinnitus Data Archive), are traumatic events, primarily the acoustic trauma $(20 \%)$, followed by head trauma (9\%); below to rate the disease processes in the middle and interior ear, especially otosclerosis and Meniere's disease (7\%) and the consumption of ototoxic drugs $(2 \%)$. In the remaining $60 \%$ of cases it is not possible to pinpoint a specific factor responsible for the onset of tinnitus. Certainly other factors play an important role, in addition to the above mentioned, such as nutritional deficiencies, endocrine and metabolic disorders, anxietydepression, and finally the temporomandibular disorders. The TMD patients complaining of tinnitus seem to have greater muscle-tendon laxity and an increased level of stress, compared to those without tinnitus. There have been a number of theories proposed to explain the association TMD-tinnitus, but still they did not gain consensus in the scientific community. More recent studies of Vielsmeier ${ }^{10,11}$, tend to go in the same direction, identifying TMD-related tinnitus as a specific subtype of tinnitus, less tied to classical risk factors such as older age and prevalence in males, which may modulate with somatic or acoustic stimuli. All of the etiopathogenetic hypotheses proposed from 1934 to the present can roughly be summarized in three basic theories:

Mechanical Theory (disc-malleolar traction): based on the debated presence of a pseudo-ligamentous connective structure present in fetal age, described by Pinto $^{12}$ as a thin ligament, able to mobilize the tympanumossicular unit as result of condylar-disk movements. Different studies have been published after that, with anatomical and biomechanical observations excluding the possibility that the fibrous structure of the TMJ can transmit motion at the joint ${ }^{13,14}$.

Myogenic theory: based on the effects of hypertonicity of the masticatory muscles, with involvement of the tensor tympani and tensor veli and the anatomical relationships between the muscles and the middle ear ${ }^{15,16}$. The overtone of the tensor tympani muscle would increase the perilymph pressure, thus affecting hearing, while vestibular overtone of the tensor veli would rather impact on the middle ear by interfering with the normal dynamics of the opening of the tube. An Italian study in 1996 conducted with the EMG did not show differences in the temporal and masseter muscle hypertonicity between two groups of TMD patients, respectively, with and without concomitant otoneurologic disorders ${ }^{17}$.

Anatomical theory: based on anatomical contiguity between auricular-temporal nerve and the condyle-disc complex, widely discussed in the literature, Cascone ${ }^{18,19}$ has recently recovered the value of the theory for potential situations of "impingement" at the anteromedial subluxation of TMJ disc, possibly at the base of atypical trigeminal symptoms. In fresh adult cadavers, the demonstration of the close anatomic relationship between the course of the auriculo-temporal nerve and anterior and medial aspect of the joint capsule of the TMJ may in fact explain the extension by contiguity of the effects of TMD on the territory of the trigeminal competence ${ }^{20}$.

The authors believe that the anatomic theory perfectly fits with that of the so- called mechanism "crossmodal" neuroplasticity. Studies in animal models have already highlighted the neural pathways through which the trigeminal afferents may interact at the level of the central nervous system ${ }^{21,22}$. The trigeminal input, through the Gasser ganglio and the trigeminal nuclear complex, reaches the dorsal cochlear nucleus $(\mathrm{DCN})^{23}$, and may affect the activity of the central sound ${ }^{24}$, especially in situations of concomitant cochlear damage. Moreover, in patients suffering from tinnitus, it has been shown that the intensity of the symptom can be modulated by a somatosensory stimulation (electrical stimulation of the median nerve at the wrist) ${ }^{24}$, an experience that is interpreted as non-specific activation of nerve structures in the process of extra-lemniscal tinnitus genesis ${ }^{25}$. Taking into account that the association tinnitus-TMD is a common condition, that many patients can modify their tinnitus through the cranio-cervical-mandibular movements, and finally, that somatosensory imput can be transmitted through a neuronal pathway to DCN, it has been proposed the concept of "somatosensory tinnitus" that may be further defined as "TMD- related somatosensory tinnitus" (TMD-RST), identified as a specific tinnitus subtype with specific characteristics and supported by a successive seexperimental evidence ${ }^{26,27}$.

\section{CONCLUSION}

The controversial aspects related to the presence of otologic symptoms in patients suffering from TMD have yet to be clarified in a definitive manner, even though as of today some of the physiopathological mechanisms underlying tinnitus genesis in conditions of TMD are known. The results of this study provide an experiential contribution that goes in the same direction of what already and widely reported in the world literature, namely, that there is a close correlation between the joint pathology and otologic symptoms, particularly regarding tinnitus and balance disorders, and that this relationship is greater more advanced is the stage of joint pathology. Moreover, unlike to what happens for the non TMD-related tinnitus, this study shows that TMDrelated tinnitus principally affects a younger population (fifth decade of life) and mainly women (more than 2/3 of the cases). Such evidence suggests the existence of a specific tinnitus subtype that may be defined as "TMDrelated somatosensory tinnitus".

\section{REFERENCES}

1. Costen JB. A syndrome of ear and sinus symptoms dependent upon disturbed function of the temporomandibular joint. 1934. Ann Otol Rhinol Laryngol. 1997;106:805-19. 
2. Moulton RE. Psychiatric consideration in maxillofacial pain. J Am Dent Assoc. 1955;51:408-14.

3. Laskin DM. Etiology of the pain-dysfunction syndrome. J Am Dent Assoc. 1969;79:147.

4. Dworkin SF, LeResche L, Von Korff M. Studying the natural history of TMD: epidemiology, perspectives on physical and psycological findings. Clinical research as the basis of clinical practice. Vig CD, Vig PS, editors. Ann Arbor: University of Michigan.1991:39-60.

5. Pekkan G, Aksoy S, Hekimoglu C, Oghan F. Comparative audiometric evaluation of temporomandibular disorder patients with otological symptoms. J Craniomaxillofac Surg. 2010;38(3):231-4.

6. Pentyala S, Mysore P, Moller D, Pentyala S, Kardovich R, Martino A, et al. Temporomandibular joint disorder and inner ear pruritus: resolution by eminectomy. J Craniofac Surg 2014;25(5):1840-2.

7. Newman CW, Jacobson GP, Spitzer JB. Development of the Tinnitus Handicap Inventory. Arch Otolaryngol Head Neck Surg.1996;122:143-8.

8. Jacobson GP, Newman CW. The development of the Dizziness Handicap Inventory, Arch Otolaryngol Head Neck Surg.1990;116:424-31.

9. Wilkes $\mathrm{CH}$. Internal derangements of the temporomandibular joint: Pathological variation. Arch Otolaryngol Head Neck Surg.1989;115(4):469-77.

10. Vielsmeier V, Kleinjung T, Strutz J, Bürgers R, Kreuzer PM, Langguth B. Tinnitus with temporomandibular joint disorders: a specific entity of tinnitus patients? Otolaryngol Head Neck Surg. 2011;145(5):748-52.

11.Vielsmeier V, Strutz J, Kleinjung T, Schecklmann M, Kreuzer PM, Landgrebe $\mathrm{M}$, et al. Temporomandibular joint disorder complaints in tinnitus: further hints for a putative tinnitus subtype. PLoS One. 2012;7(6):e38887.

12.Pinto OF. A new structure related to the temporomandibular joint and middle ear. J Prosthet Dent. 1962;12:95-103.

13. Komori E, Sugisaki M, Tanabe H, Katoh S. Discomalleolar ligament in the adult human. Cranio. 1986;4:299-305.

14. Eckerdal $O$. The petrotympanic fissure: a link connecting the tympanic cavity and the temporomandibular joint. Cranio. 1991;9(1):15-22.

15. Franz B, Anderson C. The potential role of joint injury and eustachian tube dysfunction in the genesis of secondary Ménière's disease. Int Tinnitus J. 2007;13:132-7.

16. Shehhati-Chafai-Leuwer S, Wenzel S, Bschorer R, Seedorf H, Kucinski
$\mathrm{T}$, Maier $\mathrm{H}$, et al. Pathophysiology of the Eustachian tube. Relevant new aspects for the head and neck surgeon. J Craniomaxillofac Surg. 2006;34:351-54.

17. Manni A, Brunori P, Giuliani M, Modoni M, Bizzi G. Oto-vestibular symptoms in patients with temporomandibular joint dysfunction. Electromyographic study. Minerva Stomatol. 1996;45:1-7.

18. Cascone P, Fatone FM, Paparo F, Arangio P, lannetti G. Trigeminal impingement syndrome: the relationship between atypical trigeminal symptoms and anteromedial disk displacement. Cranio. 2010;28(3):177-80.

19.Attanasio G, Leonardi A, Arangio P, Minni A, Covelli E, Pucci R, et al. Tinnitus in patients with temporo-mandibular joint disorder: Proposal for a new treatment protocol. J Craniomaxillofac Surg. 2015;43(5):724-7.

20.Paparo F, Fatone FMG, Ramieri V, Cascone P. Anatomic relationship between trigeminal nerve and temporomandibular joint. Eur Rev Med Pharmacol Sci. 2008;12:15-8.

21. Roberts LE, Eggermont JJ, Caspary DM, Shore SE, Melcher JR, Kaltenbach JA. Ringing ears: the neuroscience of tinnitus. J Neurosci. 2010;30:14972-79.

22.Dehmel S, Pradhan S, Koeheler S, Bledsoe S, Shore S. Noise overexposure alters long- term somatosensory-auditory processing in the dorsal cochlear nucleus- possible basis for tinnitus-related hyperactivity? J Neurosci. 2012; 32:1660-71.

23. Filipo R, Attanasio G, Cagnoni L, Masci E, Russo FY, Cartocci G, et al. Long-term results of intratympanic prednisolone injection in patients with idiopathic sudden sensorineural hearing loss. Acta Otolaryngol. 2013;133(9):900-4.

24. Møller AR, Moller MB, Yokota M. Some forms of tinnitus may involve the extralemniscal auditory pathway. Laryngoscope. 1992;102:1165-71.

25. Møller AR. The role of neural plasticity in tinnitus. Progr Brain Res. 2007; 166:37-45.

26. Vanneste S, Plazier M, der Loo E. The neural correlates of tinnitusrelated distress. Neuroimage. 2010;52:470-80.

27.Kapoula Z, Yang Q, Le TT, Vernet M, Berbey N, Orssaud C, et al. Medio-lateral postural instability in subjects with tinnitus. Front Neurol. 2011;2:35. 